\title{
BMI, functional and cognitive status in a cohort of nonagenarians: results from the Mugello study
}

\author{
Monica Dinu ${ }^{1}$ (1) - Barbara Colombini ${ }^{1}$. Giuditta Pagliai ${ }^{1} \cdot$ Federica Vannetti ${ }^{2}$. Guido Pasquini ${ }^{2}$. \\ Raffaello Molino Lova ${ }^{2}$. Francesca Cecchi ${ }^{2}$. Sandro Sorbi ${ }^{2}$. Francesco Sof ${ }^{1,2}$. Claudio Macchi ${ }^{2}$. The Mugello Study \\ Working Group
}

Received: 28 July 2020 / Accepted: 7 October 2020 / Published online: 21 October 2020

(c) The Author(s) 2020

\section{Key summary points}

Aim Our aim was to investigate the association of body mass index (BMI) with functional and cognitive status in a group of nonagenarians.

Findings By grouping the participants according to BMI categories, overweight and obese participants showed lower functional capability, higher risk of falling but better Mini-Mental State Examination (MMSE) performance than participants with normal weight or underweight.

Message This cross-sectional survey supports the hypothesis that adiposity could affect the cognitive state of people reaching the old age.

\begin{abstract}
Purpose The study of the relationship between body weight and health in old age has attracted increasing interest. The aim of the present study is to investigate the association of body mass index (BMI) with functional and cognitive status in a group of nonagenarians.

Methods We analyzed 475 participants (348 women, 127 men; median age 92 years) from the Mugello study. Participants were evaluated through laboratory, instrumental examinations and questionnaires.

Results By grouping the participants according to BMI categories, a better perception of health and nutritional status and a lower prevalence of sarcopenia $(p<0.05)$ were observed in participants with overweight and obesity compared to participants with normal weight or underweight. Concerning functional and cognitive measures, overweight and obese participants showed significantly worse performance on short physical performance battery and timed up and go tests and better performance on the mini-mental state examination (MMSE). As regards the other tests performed, no statistically significant differences were observed. In a multivariate logistic regression analysis adjusted for possible confounding factors, participants with BMI $\geq 30 \mathrm{~kg} /$ $\mathrm{m}^{2}$ showed lower probability to achieve poor performance on the MMSE (OR 0.42; 95\% CI 0.19-0.94; $p=0.035$ ).

Conclusion Our results support the hypothesis that in nonagenarians, a higher BMI is associated with better cognitive ability. Further studies are needed to explore the mechanisms underlying this association.
\end{abstract}

Keywords Cognitive status $\cdot$ Functional status $\cdot$ Nonagenarians $\cdot$ Obesity paradox $\cdot$ BMI

The members of The Mugello Study Working Group are listed in acknowledgements.

Monica Dinu

monica.dinu@unifi.it

1 Department of Experimental and Clinical Medicine, University of Florence, Largo Brambilla 3, 50134 Florence, Italy

2 Don Carlo Gnocchi Foundation Florence, Onlus IRCCS, Florence, Italy

\section{Introduction}

In recent years, many studies have linked functional and cognitive status to late life and body mass index (BMI) [1-3]. In terms of physical performance, a higher BMI has shown a controversial association with the effects on health and quality of life. Indeed, some studies have reported better physical functioning in older people with higher BMI [1], while others have reported more functional limitations and 
disabilities in those with higher BMI [4]. The same kind of results has been found in the literature between adiposity and cognitive state. An analysis of 2684 participants aged 65-94 [5] revealed a non-linear relationship between adiposity and cognitive function with overweight participants having better cognitive function, while Skinner et al. [6] reported significantly better cognitive flexibility performance in obese older adults.

The controversial association between adiposity and cognitive function has often been explained in the context of the "obesity paradox", a hypothesis based on the fact that in old age obesity can have protective effects against certain conditions such as cognitive impairment [7]. The mechanisms underlying the "obesity paradox", however, remain unclear, and not all the studies provide evidence to support this hypothesis $[8,9]$. Moreover, there is little evidence on the relationship between BMI, functional and cognitive status in older populations.

The aim of this paper was to investigate the association of BMI with functional and cognitive status in old age, examining data from the Mugello study, a survey of people aged 90 years and older living in the Mugello area in Tuscany, Italy.

\section{Material and methods}

\section{Study population}

The analysis presented in this paper is based on data obtained from 475 nonagenarians (348 women, 127 men) enrolled within the Mugello study. The Mugello study is a cross-sectional survey of people aged 90 years and more, living in the Mugello area (north-east of Florence, Tuscany, Italy). The study protocol has been described in detail elsewhere [10]. Briefly, for each municipality of the Mugello area, nonagenarian residents and their general practitioners were contacted by letter and then by phone to ascertain their availability to participate in the study and to schedule a visit. The sample size ( $82 \%$ of all the nonagenarians living in the area) was highly representative of this population.

\section{Data collection}

Participants were interviewed and examined at their home/ nursing home by a trained physician, following a standardized protocol. General information on demographics, education, lifestyle, dietary habits, medical history, drug use and functional and cognitive status were collected from each participant. Level of physical activity was scored by administering a questionnaire modeled on the Harvard Alumni Questionnaire and adapted for the Italian population, as described elsewhere [10]. Weight and height were measured using a stadiometer. BMI was calculated as the weight $(\mathrm{kg}) /$ height $\left(\mathrm{m}^{2}\right)$. Participants were classified as underweight if their BMI was $<18.5$, normal weight if their BMI was 18.5-24.99, overweight if their BMI was 25-29.99, and obese if their BMI was 30 or more. Body composition was determined by a bioelectrical impedance analysis device (TANITA, model TBF-410).

The study was conducted in accordance with the principles of the Declaration of Helsinki on clinical research involving human beings and was approved by the Ethical Committee of the Don Gnocchi Foundation. Informed written consent was obtained from all participants, or their legal representative.

\section{Measures}

Physical performance was evaluated using the Short Physical Performance Battery (SPPB) which assesses walking speed, standing balance and ability to raise from a chair. The total score ranges from 0 to 12 , with higher score indicating better performance. Mobility was assessed by the timed up and go test (TUG). Patients were asked to rise from a $45 \mathrm{~cm}$-high chair, walk forward $3 \mathrm{~m}$ at their usual walking pace, turn and walk back to the chair and sit down again. The score ranges from 0 to 3 , with 0 indicating falls risk, 1 need assistance, 2 normal limits and 3 normal mobility. The maximal isometric handgrip strength (HGS, $\mathrm{kg}$ ) was measured with a hydraulic dynamometer (RO+TEN, Verano Brianza, Italy). Participants repeated the test twice for each hand, and the best value for each side was recorded. The mean value of the best right and left results was used. Gait speed and functional mobility was evaluated through the Gait-speed test which measures time taken to walk $4 \mathrm{~m}$. Gait speed of longer than $5 \mathrm{~s}$ to walk $4 \mathrm{~m}(\leq 0.8 \mathrm{~m} / \mathrm{s})$ suggests an increased risk of frailty. Performance of lower extremity muscles was measured using the sit-to-stand test. Participants were asked to stand up and sit down 5 times, as quickly as they could without any form of assistance. The time to complete the test has been recorded. Functional disability was assessed by the Basic Activities of Daily Living (BADLs) [11] and the Instrumental Activities of Daily Living (IADLs) [12] tools. Participants were classified as independent if they reported a score of $0-1$ for all BADLs (eating, showering, dressing, transferring from bed to chair, using the toilet, and continence) and IADLs activities (shopping, doing light housework, preparing meals, managing money, using the telephone, taking medications, using transportation, and doing laundry), corresponding to perform all the considered activities without the help of another person.

The mini-mental state examination (MMSE) was used to tests 5 areas of cognitive function: orientation, registration, attention and calculation, recall and language [13]. The global score ranges from 0 to 30 , with a score of 23 or less 
indicating severe ( $\leq 9$ points), moderate (10-18 points) or mild (19-23) dementia. Depressive symptoms were investigated by the 15-item Geriatric Depression Scale (GDS) [14]. Scores of 0-4 were considered normal. Quality of life was assessed by the 12-Item Short-Form Health Survey (SF12). Physical (PCS) and Mental (MCS) components were considered separately.

\section{Definition of sarcopenia}

Sarcopenia was evaluated based on the diagnostic criteria of the European Working Group on Sarcopenia in Older People updated recommendations (EWGSOP2) [15]. Low muscle strength was defined as handgrip strength $<27 \mathrm{~kg}$ in men and $<16 \mathrm{~kg}$ in women. Low muscle quantity and quality was defined as appendicular skeletal muscle mass $(\mathrm{ASM})<20 \mathrm{~kg}$ in men and $<15 \mathrm{~kg}$ in women. Raw measures produced by bioelectrical impedance analysis were used for estimation of ASM mass, using the cross-validated Sergi equation for standardization [16]. Low physical performance was defined as SPPB $\leq 8$-point score. Individuals with low muscle strength plus low muscle quantity and quality or low physical performance were categorized as sarcopenic.

\section{Statistical analysis}

The statistical package PASW 20.0 for Macintosh (SPSS Inc., Chicago, IL, US) was utilized. Data were reported as mean \pm standard deviation (SD), median and range or number and percentage, as appropriate. To analyze the possible relationship between BMI, functional and cognitive status, participants were grouped according to BMI categories. The Kruskal-Wallis test was used for comparisons among different groups. The Pearson's Chi-square was used to test for proportions.

The association between BMI categories, functional and cognitive status was assessed using logistic regression analysis, with normal weight participants as reference group. Variables that were shown to be significantly different among groups in the descriptive analysis were introduced in a univariate model. A score $\leq 8$ for SPPB,$<1$ for TUG and $\leq 23$ for MMSE was used as independent variable and BMI categories were used as dependent variables. A multivariate model was then performed to evaluate the associations after adjustment for possible confounding factors such as age, gender, marital status (widowed, married/ partner, single/divorced), education (no diploma, elementary school, middle school, $\geq$ high school), smoking (yes, no), physical activity (more than once a week, no), waist-to-hip ratio (WHR), depression (GDS $\leq 4,>4$ ), sarcopenia (yes, no) and comorbidities (at least one of hypertension, diabetes, cardiovascular disease, and/or cerebrovascular disease, no).
Results were reported as odds ratio (OR) and 95\% confidence intervals (CI). Values were considered significant at $p<0.05$.

\section{Results}

The characteristics of the study population according to body weight status are reported in Table 1 . Of the 475 participants, most were women $(73.3 \%)$, and the median age was 92 years. Significant $(p<0.05)$ differences among BMI categories were observed for self-perception of general health and nutritional status, anthropometric parameters and prevalence of sarcopenia. Participants with overweight and obesity had better perception of their health and nutritional status, higher body fat mass, muscle mass and waist-hip ratio, and reported lower prevalence rates of sarcopenia compared with participants with lower BMI.

Mean scores of physical and cognitive function tests according to body weight status are reported in Table 2 . With regard to functional measures, significant differences among BMI categories were observed for SPPB $(p=0.005)$ and TUG $(p=0.010)$ tests. Participants classified as overweight and obese reported worse performance on both tests, denoting lower functional capability and higher risk of falling. With regard to cognitive tests, a significant difference among BMI categories was reported for MMSE $(p=0.003)$, with better performance in overweight and obese participants. No significant differences were observed for the other tests performed. By evaluating the functional independence, obese participants showed significantly $(p=0.004)$ lower functional independence $(n=14 ; 21.9 \%)$ compared with normal weight $(n=68 ; 30 \%)$ and underweight $(n=11 ; 40.7 \%)$ participants.

Finally, the relationship between BMI categories, physical and cognitive tests was assessed using logistic regression analysis. As reported in Table 3, participants with $\mathrm{BMI} \geq 30 \mathrm{~kg} / \mathrm{m}^{2}$ reported significantly higher risk of functional impairment (SPPB $\leq 8$ points) (OR 4.47; 95\% CI $1.30-15.33 ; p=0.017)$ and falls (TUG $<1$ point) (OR $2.58 ; 95 \%$ CI $1.15-5.79 ; p=0.022$ ), and lower probability to achieve poor performance on the MMSE ( $\leq 23$ points) (OR $0.50 ; 95 \%$ CI $0.28-0.87 ; p=0.015$ ). After adjustment for possible confounding factors such as age, gender, marital status, education, smoking, physical activity, waist-hip ratio, depression, sarcopenia and comorbidities, the associations with functional impairment and falls risk lost their statistical significance. As regards the MMSE performance, the association remained statistically significant (OR $0.42 ; 95 \%$ CI $0.19-0.94 ; p=0.035$ ) in participants with $\mathrm{BMI} \geq 30 \mathrm{~kg} / \mathrm{m}^{2}$. 
Table 1 Characteristics of study population according to BMI categories

\begin{tabular}{|c|c|c|c|c|c|}
\hline & $\begin{array}{l}\text { Underweight } \\
n=27\end{array}$ & $\begin{array}{l}\text { Normal weight } \\
n=227\end{array}$ & $\begin{array}{l}\text { Overweight } \\
n=157\end{array}$ & $\begin{array}{l}\text { Obese } \\
n=64\end{array}$ & $p$ value* \\
\hline Age, years & $93(90-106)$ & $92(88-105)$ & $92(89-103)$ & $91(89-102)$ & 0.311 \\
\hline Women, $n(\%)$ & $24(88.9)$ & $167(73.6)$ & $108(68.8)$ & $49(76.6)$ & 0.149 \\
\hline \multicolumn{6}{|l|}{ Marital status } \\
\hline Widowed, $n(\%)$ & $20(74.1)$ & $164(72.2)$ & $126(80.3)$ & 55 (85.9) & \multirow[t]{3}{*}{0.310} \\
\hline Married/partner, $n(\%)$ & $5(18.5)$ & 49 (21.6) & 25 (15.9) & $7(10.9)$ & \\
\hline Single/divorced, $n(\%)$ & $2(7.4)$ & $14(6.2)$ & $6(3.8)$ & $2(3.1)$ & \\
\hline Living alone, $n(\%)$ & $4(14.8)$ & $34(15)$ & $36(22.9)$ & $10(15.6)$ & 0.227 \\
\hline Education, years & $3.9 \pm 3.1$ & $4.3 \pm 3.1$ & $4.4 \pm 2.9$ & $3.8 \pm 2.2$ & 0.206 \\
\hline \multicolumn{6}{|l|}{ Smoking status } \\
\hline Current, $n(\%)$ & $1(3.7)$ & $7(3.1)$ & $2(1.3)$ & - & \multirow[t]{2}{*}{0.374} \\
\hline Former, $n(\%)$ & $4(14.8)$ & $63(27.8)$ & $48(30.6)$ & $16(25)$ & \\
\hline Absent physical activity, $n(\%)$ & $13(48.1)$ & $115(50.7)$ & $83(52.9)$ & $40(62.5)$ & 0.497 \\
\hline \multicolumn{6}{|l|}{ Health compared with peers ${ }^{a}$} \\
\hline Better, $n(\%)$ & $7(25.9)$ & $86(37.9)$ & $75(47.8)$ & $25(39.1)$ & \multirow[t]{2}{*}{0.033} \\
\hline Worse, $n(\%)$ & $3(11.1)$ & $22(9.7)$ & $7(4.5)$ & $4(6.3)$ & \\
\hline Good nutritional status ${ }^{\mathrm{a}}, n(\%)$ & $12(44.4)$ & $165(72.7)$ & $132(84.6)$ & $60(93.8)$ & $<0.001$ \\
\hline \multicolumn{6}{|l|}{ Anthropometric parameters } \\
\hline Fat mass, $\%$ & $11.2 \pm 8.0$ & $19.8 \pm 9.7$ & $27.8 \pm 10.6$ & $34.3 \pm 10$ & $<0.001$ \\
\hline Muscle mass, $\mathrm{kg}$ & $23.7 \pm 5.8$ & $28.8 \pm 8.5$ & $31.1 \pm 9.0$ & $32.9 \pm 6.6$ & $<0.001$ \\
\hline Waist-hip ratio & $0.93 \pm 0.15$ & $0.94 \pm 0.09$ & $0.95 \pm 0.08$ & $0.95 \pm 0.07$ & 0.031 \\
\hline \multicolumn{6}{|l|}{ Comorbidities and medications } \\
\hline Cardiovascular diseases, $n(\%)$ & $9(33.3)$ & $96(42.3)$ & $71(45.2)$ & $25(39.1)$ & 0.533 \\
\hline Cerebrovascular diseases, $n(\%)$ & $4(14.8)$ & $41(18.1)$ & $36(22.9)$ & $15(23.4)$ & 0.453 \\
\hline Diabetes, $n(\%)$ & $1(3.7)$ & $30(13.2)$ & $26(16.6)$ & $11(17.2)$ & 0.282 \\
\hline Hypertension, $n(\%)$ & $11(40.7)$ & $96(42.3)$ & $82(52.2)$ & $34(53.1)$ & 0.162 \\
\hline Sarcopenia, $n(\%)$ & $14(51.9)$ & $85(37.4)$ & $42(26.8)$ & $7(10.9)$ & $<0.001$ \\
\hline No of medications & $3.3 \pm 1.8$ & $3.3 \pm 2$ & $3.8 \pm 2$ & $3.5 \pm 2$ & 0.133 \\
\hline
\end{tabular}

Continuous variables are expressed as mean $\pm \mathrm{SD}$ or as median (range). Categorical variables are expressed as number (percentage)

* $p$ values calculated using Kruskal-Wallis test or $\chi^{2}$ test

${ }^{\text {a }}$ Self-perceived

\section{Discussion}

The relationship between BMI and health in old age has been a controversial issue for many years. By grouping a large cohort of nonagenarians according to BMI categories, in the present paper we were able to report that overweight and obese participants had a lower functional capability, a higher risk of falling but better MMSE performance than participants with normal weight or underweight, thus supporting the hypothesis that adiposity could affect the cognitive state of people reaching the old age.

To date, the interaction between body weight, adiposity and cognitive function in the ninth decade of life is poorly understood [17]. Cognition is an extremely complex activity influenced by a multiplicity of biological cerebral mechanisms [18], and the comorbidities associated with overweight and obesity such as diabetes, cardiovascular disease, hypertension and sleep apnoea syndrome may explain most of the association between BMI and increased risk of cognitive impairment [19]. In older people, on the other hand, several studies suggested that the direction of the relationship between BMI and cognitive impairment may change in a counterintuitive way [20]. The negative effect of high BMI on cognitive functions in older people is less clear than in young adults and middle-aged people [21], and both positive and negative associations have been reported from studies on BMI in later life and dementia risk [6, 22].

In the present study, nonagenarians with a high BMI, without reporting a significant prevalence of sarcopenia and despite having a higher risk of falling and of functional disabilities, presented better MMSE scores. These results are in line with several papers reporting the so-called "obesity paradox" hypothesis [22, 23], that is a reduced risk of cognitive decline associated with overweight in late life, even if 
Table 2 Physical and cognitive function scores according to BMI categories
Table 3 Logistic regression analysis for the association between BMI categories, functional and cognitive tests

\begin{tabular}{lccccc}
\hline & Underweight & Normal weight & Overweight & Obese & $p$ value* \\
\hline Physical function & & & & & \\
SPPB (0-12 points) & $4.2 \pm 3.5$ & $4.5 \pm 2.9$ & $3.9 \pm 2.6$ & $2.9 \pm 2.2$ & 0.005 \\
TUG (0-3 points) & $1.1 \pm 1$ & $1.5 \pm 0.8$ & $1.2 \pm 0.8$ & $1.1 \pm 0.9$ & 0.010 \\
HGS test & $11.6 \pm 5.8$ & $15.0 \pm 7.5$ & $14.9 \pm 7.0$ & $14.4 \pm 5.8$ & 0.155 \\
Gait-speed test, m/s & $0.5 \pm 0.3$ & $0.5 \pm 0.3$ & $0.4 \pm 0.3$ & $0.4 \pm 0.2$ & 0.315 \\
Sit-to-stand test, s & $24.4 \pm 16.1$ & $21.3 \pm 13.1$ & $21.7 \pm 11.4$ & $23.7 \pm 15.0$ & 0.752 \\
BADLs (0-6 points) & $3 \pm 2.5$ & $3.3 \pm 2.3$ & $3.5 \pm 2$ & $3.2 \pm 1.9$ & 0.700 \\
IADLs (0-8 points) & $3.2 \pm 3.3$ & $3.8 \pm 3.4$ & $3.9 \pm 3.2$ & $4 \pm 3$ & 0.668 \\
PCS (0-100 points) & $43.4 \pm 8.6$ & $43.6 \pm 8.1$ & $42.6 \pm 7.9$ & $41 \pm 7$ & 0.213 \\
Cognitive function & & & & & \\
MMSE (0-30 points) & $14.5 \pm 9.7$ & $18.4 \pm 10$ & $19.7 \pm 8.8$ & $21.9 \pm 7.7$ & 0.003 \\
MCS (0-100 points) & $45 \pm 6.3$ & $47.7 \pm 7.5$ & $47.4 \pm 6.9$ & $46.7 \pm 7.1$ & 0.644 \\
GDS (0-15 points) & $6.3 \pm 4.6$ & $4.4 \pm 3.7$ & $5.1 \pm 3.9$ & $4.2 \pm 4$ & 0.104 \\
\hline
\end{tabular}

Data are expressed as mean $\pm \mathrm{SD}$

$S P P B$ short physical performance battery, $T U G$ timed up and go, $H G S$ hand grip strength, $B A D L$ basic activities of daily living, IADL instrumental activities of daily living, PCS physical component summary scores, MMSE mini-mental state examination, MCS mental component summary scores, GDS geriatric depression score

* $p$ values calculated using Kruskal-Wallis test

\begin{tabular}{|c|c|c|c|c|c|c|}
\hline \multirow[t]{2}{*}{ Variable } & \multicolumn{3}{|c|}{ Univariate analysis } & \multicolumn{3}{|c|}{ Multivariate analysis $^{\mathrm{a}}$} \\
\hline & $\mathrm{OR}$ & $95 \% \mathrm{CI}$ & $p$ value & $\overline{\mathrm{OR}}$ & $95 \% \mathrm{CI}$ & $p$ value \\
\hline \multicolumn{7}{|l|}{$\mathrm{SPPB} \leq 8$} \\
\hline Underweight & 0.04 & $0.27-4.00$ & 0.955 & 1.94 & $0.28-13.36$ & 0.500 \\
\hline Normal weight & & Reference & & & Reference & \\
\hline Overweight & 1.76 & $0.93-3.35$ & 0.085 & 1.55 & $0.71-3.37$ & 0.274 \\
\hline Obese & 4.47 & $1.30-15.33$ & 0.017 & 3.43 & $0.89-13.20$ & 0.073 \\
\hline \multicolumn{7}{|l|}{ TUG $<1$} \\
\hline Underweight & 3.19 & $0.97-10.52$ & 0.056 & 5.97 & $1.27-28.14$ & 0.024 \\
\hline Normal weight & & Reference & & & Reference & \\
\hline Overweight & 1.82 & $0.95-3.49$ & 0.069 & 1.55 & $0.71-3.39$ & 0.274 \\
\hline Obese & 2.58 & $1.15-5.79$ & 0.022 & 1.69 & $0.64-4.44$ & 0.291 \\
\hline \multicolumn{7}{|l|}{$\mathrm{MMSE} \leq 23$} \\
\hline Underweight & 1.37 & $0.59-3.21$ & 0.706 & 0.80 & $0.26-2.52$ & 0.555 \\
\hline Normal weight & & Reference & & & Reference & \\
\hline Overweight & 0.93 & $0.61-1.40$ & 0.710 & 1.18 & $0.67-2.08$ & 0.572 \\
\hline Obese & 0.50 & $0.28-0.87$ & 0.015 & 0.42 & $0.19-0.94$ & 0.035 \\
\hline
\end{tabular}

$S P P B$ short physical performance battery, $T U G$ timed up and go, MMSE mini-mental state examination

${ }^{a}$ Adjusted for age, gender, marital status (widowed, married/partner, single/divorced), education (no diploma, elementary school, middle school, $\geq$ high school), smoking (yes, no), physical activity (more than once a week, no), waist-hip ratio, depression (GDS $\leq 4,>4$ ), sarcopenia (yes, no) and comorbidities (at least one of hypertension, diabetes, cardiovascular disease, and/or cerebrovascular disease, no) the issue remain controversial [24]. A very recent observational study investigating the relationship between obesity and cognitive impairment in 1100 patients aged 60-98 years has shown that overweight was associated with a lower risk of cognitive impairment [25], and the results of a prospective study revealed that older people with higher BMI had a lower risk of dementia than their counterparts with lower BMI [26]. On the other hand, other studies found no association between BMI and dementia [27], and several data showed an increased risk of cognitive impairment with a higher BMI in mid-life, reporting a negative effect of body weight gain on cognitive function in old age [21]. 
Therefore, the possible mechanisms linking adiposity and improved cognitive function in older age remain unclear, leaving room for speculation. One possible hypothesis is the "survival effect". This hypothesis suggests that people who are susceptible to the negative effects of obesity die earlier, and those who survive into old age may be an obese subgroup resistant to such effects [28]. Thus, late-life overweight and obesity may reflect survivors with protective characteristics against vascular risk factors and vascular dementia. Another hypothesis is that overweight or obese older people may have a better intake of trace elements, vitamins, and other nutrients that improve the functioning of the molecular pathways involved in the regulation of cognitive abilities [28]. A further intrinsic mechanism could be through leptin, a hormone mainly secreted by adipose tissue, that has been shown to improve learning and memory performance in animal models by regulating the synaptic plasticity of the hippocampus and the beta-process of amyloid [29], and whose high levels were associated with lower risk of dementia [30].

A clinical aspect that can mediate the possible link between body weight and cognitive state is the presence of sarcopenia [16]. Sarcopenia has been widely reported to be associated with poor functional performance in older people [31] and has been shown to be probably associated with cognitive impairment [32]. In the population covered by this study, we have not observed a high prevalence of sarcopenia, and this can be probably explained by the fact that most of the nonagenarians in our population continued to live at home, alone or with their family [10]. As already reported in a previous article from the same project, the Mugello study included a large cohort of nonagenarians living in small villages where the social community continues to support older people not to be isolated and not to live in clinics [10]. This may have influenced positively the low prevalence of sarcopenia and helps adding another hypothesis on the relationship between body weight and cognitive function since BMI, in non-sarcopenic older people, may represent a strong predictor of skeletal muscle mass that seems positively associated with improved cognitive ability [33].

The present study has several strengths and some limitations that deserve comments. The Mugello study offered many advantages to investigate the association between BMI, functional and cognitive status in older people through objective measurements of anthropometric parameters and validated tests of functioning and cognitive capacity. Indeed, only a few studies have explored the relationship between BMI and cognitive status in the older healthy population and even rarer are the data referring to the relationship between BMI, functional and cognitive status in nonagenarian groups. However, this analysis come from a cross-sectional survey that precludes the possibility of establishing causal relationships. Since we do not know the past weight of the participants in middle age, we cannot therefore evaluate the trajectory of obesity in our analysis and assess how long- or short-term obesity has affected the functional and cognitive state in old age. In addition, the population of our study that reported a normal body weight included not only those who had always been thin, but also those who lost weight unintentionally due to diseases related to the general decline in health and the development of cognitive impairment. In older people low BMI is usually a consequence of malnutrition, dysphagia, and masticatory dysfunction or bowel disorders $[28,34]$.

In conclusion, this cross-sectional survey from the Mugello study showed that a higher BMI is associated with lower functional capability but with better cognitive ability, supporting the hypothesis that obesity may be a protective factor against cognitive impairment in nonagenarians according to "obesity paradox". Evidence of this paradox confirms that the risk relationship between BMI and cognitive status can change over the course of life from mid-life to late life, suggesting that optimal BMI goals in older people have yet to be established. Further studies are needed to better understand the general health status of nonagenarians and to identify strategies for its improvement.

Acknowledgements The Mugello Study Working Group also includes Roberta Boni, Chiara Castagnoli, Annamaria Gori, Anita Paperini, Lorenzo Razzolini, Nona Turcan, Debora Valecchi, Gianfranco Gensini, and Rosanna Abbate.

Author contributions Conception and design: MD, BC, FS, CM; analysis and interpretation of the data: MD, BC, GiP, FS; drafting of the article: MD, BC, GiP, FS, CM; clinical evaluation of patients: GuP, FC; critical revision of the article for important intellectual content: SS, CM; final approval of the article: FS, CM; statistical expertise: FV, CML, FS.

Funding Open access funding provided by Università degli Studi di Firenze within the CRUI-CARE Agreement. This work was supported by Current Research Funds (2014/2015) from the Italian Ministry of Health.

\section{Compliance with ethical standards}

Conflict of interest On behalf of all authors, the corresponding author states that there is no conflict of interest.

Ethics approval Study protocol was approved by the Ethical Committee of the Don Gnocchi Foundation.

Consent to participate Informed written consent was obtained from all participants, or their legal representative.

Consent for publication Patients signed informed consent regarding publishing their data.

Availability of data and material On request. 
Open Access This article is licensed under a Creative Commons Attribution 4.0 International License, which permits use, sharing, adaptation, distribution and reproduction in any medium or format, as long as you give appropriate credit to the original author(s) and the source, provide a link to the Creative Commons licence, and indicate if changes were made. The images or other third party material in this article are included in the article's Creative Commons licence, unless indicated otherwise in a credit line to the material. If material is not included in the article's Creative Commons licence and your intended use is not permitted by statutory regulation or exceeds the permitted use, you will need to obtain permission directly from the copyright holder. To view a copy of this licence, visit http://creativecommons.org/licenses/by/4.0/.

\section{References}

1. Bahat G, Tufan F, Saka B, Akin S, Ozkaya H, Yucel N, Erten N, Karan MA (2012) Which body mass index (BMI) is better in the elderly for functional status? Arch Gerontol Geriatr 54:78-81

2. Qizilbash N, Gregson J, Johnson ME, Pearce N, Douglas I, Wing K, Evans SJW, Pocock SJ (2015) BMI and risk of dementia in two million people over two decades: a retrospective cohort study. Lancet Diabetes Endocrinol 3:431-436

3. Pedditzi E, Peters R, Beckett N (2016) The risk of overweight/ obesity in mid-life and late life for the development of dementia: a systematic review and meta-analysis of longitudinal studies. Age Ageing 45:14-21

4. Al Snih S, Ottenbacher KJ, Markides KS, Kuo YF, Eschbach K, Goodwin JS (2007) The effect of obesity on disability vs mortality in older Americans. Arch Intern Med 167:774-780

5. Kuo HK, Jones RN, Milberg WP, Tennstedt S, Talbot L, Morris JN, Lipsitz LA (2006) Cognitive function in normal-weight, overweight, and obese older adults: an analysis of the advanced cognitive training for independent and vita elderly cohort. J Am Geriatr Soc 54:97-103

6. Skinner JS, Abel WM, McCoy K, Wilkins CH (2017) Exploring the "Obesity Paradox" as a correlate of cognitive and physical function in community-dwelling black and white older adults. Ethn Dis 27:387-394

7. Hsu CL, Voss MW, Best JR, Handy TC, Madden K, Bolandzadeh N, Liu-Ambrose T (2015) Elevated body mass index and maintenance of cognitive function in late life: exploring underlying neural mechanisms. Front Aging Neurosci 7:155

8. Strandberg TE, Stenholm S, Strandberg AY, Salomaa VV, Pitkälä KH, Tilvis RS (2013) The "obesity paradox", frailty, disability, and mortality in older men: a prospective, longitudinal cohort study. Am J Epidemiol 178:1452-1460

9. Aslan AK, Starr JM, Pattie A, Deary I (2015) Cognitive consequences of overweight and obesity in the ninth decade of life? Age Ageing 44:59-65

10. Molino-Lova R, Sofi F, Pasquini G, Gori A, Vannetti F, Abbate R, Gensini GF, Macchi C, Mugello Study Working Group (2013) The Mugello study, a survey of nonagenarians living in Tuscany: design, methods and participants' general characteristics. Eur J Intern Med 24:745-749

11. Katz S, Ford AB, Moskowitz RW, Jackson BA, Jaffe MW (1963) Studies of illness in the aged. The index of ADL: a standardized measure of biological and psychosocial function. JAMA 185:914-919

12. Lawton MP, Brody EM (1969) Assessment of older people: selfmaintaining and instrumental activities of daily living. Gerontologist 9:179-186
13. Folstein MF, Folstein SE, McHugh PR (1975) "Mini-mental state". A practical method for grading the cognitive state of patients for the clinician. J Psychiatr Res 12:189-198

14. Yesavage JA, Brink TL, Rose TL, Lum O, Huang V, Adey M, Leirer VO (1982) Development and validation of a geriatric depression screening scale: a preliminary report. J Psychiatr Res 17:37-49

15. Sergi G, De Rui M, Veronese N, Bolzetta F, Berton L, Carraro S, Bano G, Coin A, Manzato E, Perissinotto E (2015) Assessing appendicular skeletal muscle mass with bioelectrical impedance analysis in free-living Caucasian older adults. Clin Nutr 34:667-673

16. Cruz-Jentoft AJ, Bahat $\mathrm{G}$, Bauer J, Boirie $\mathrm{Y}$, Bruyère $\mathrm{O}$, Cederholm T, Cooper C, Landi F, Rolland Y, Sayer AA, Schneider SM, Sieber CC, Topinkova E, Vandewoude M, Visser M, Zamboni M, Writing Group for the European Working Group on Sarcopenia in Older People 2 (EWGSOP2), and the Extended Group for EWGSOP2 (2019) Sarcopenia: revised European consensus on definition and diagnosis. Age Ageing 48:16-31

17. García-Ptacek S, Faxén-Irving G, Cermáková P, Eriksdotter M, Religa D (2014) Body mass index in dementia. Eur J Clin Nutr 68:1204-1209

18. Fielding RA, Gunstad J, Gustafson DR, Heymsfield SB, Kral JG, Launer LJ, Penninger J, Phillips DI, Scarmeas N (2013) The paradox of overnutrition in aging and cognition. Ann N Y Acad Sci 1287:31-43

19. Cheng G, Huang C, Deng H, Wang H (2012) Diabetes as a risk factor for dementia and mild cognitive impairment: a meta-analysis of longitudinal studies. Intern Med J 42:484-491

20. Emmerzaal TL, Kiliaan AJ, Gustafson DR (2015) 2003-2013: a decade of body mass index, Alzheimer's disease, and dementia. J Alzheimers Dis 43:739-755

21. Anstey KJ, Cherbuin N, Budge M, Young J (2011) Body mass index in midlife and late-life as a risk factor for dementia: a metaanalysis of prospective studies. Obes Rev 12:e426-e437

22. Puzianowska-Kuznicka M, Kuryłowicz A, Walkiewicz D, Borkowska J, Owczarz M, Olszanecka-Glinianowicz M, Wieczorowska-Tobis K, Skalska A, Szybalska A, Mossakowska M (2019) Obesity paradox in Caucasian seniors: results of the PolSenior Study. J Nutr Health Aging 23:796-804

23. Pegueroles J, Jiménez A, Vilaplana E, Montal V, Carmona-Iragui M, Pané A, Alcolea D, Videla L, Casajoana A, Clarimón J, Ortega E, Vidal J, Blesa R, Lleó A, Fortea J, Alzheimer's Disease Neuroimaging Initiative (2018) Obesity and Alzheimer's disease, does the obesity paradox really exist? A magnetic resonance imaging study. Oncotarget 9:34691-34698

24. Dahl AK, Löppönen M, Isoaho R, Berg S, Kivelä SL (2008) Overweight and obesity in old age are not associated with greater dementia risk. J Am Geriatr Soc 56:2261-2266

25. Atti AR, Palmer K, Volpato S, Winblad B, De Ronchi D, Fratiglioni L (2010) Late-life body mass index and dementia incidence: nine-year follow-up data from the Kungsholmen Project. J Am Geriatr Soc 56:111-116

26. Tolppanen AM, Ngandu T, Kåreholt I, Laatikainen T, Rusanen M, Soininen H, Kivipelto M (2013) Midlife and late-life body mass index and late-life dementia: results from a prospective population-based cohort. J Alzheimers Dis 38:201-209

27. Inelmen EM, Sergi G, Coin A, Miotto F, Peruzza S, Enzi G (2003) Can obesity be a risk factor in elderly people? Obes Rev 4:147-155

28. Kane RL, Shamliyan T, Talley K, Pacala J (2012) The association between geriatric syndromes and survival. J Am Geriatr Soc 60:896-904 
29. Oomura Y, Hori N, Shiraishi T, Fukunaga K, Takeda H, Tsuji M, Matsumiya T, Ishibashi M, Aou S, Li XL, Kohno D, Uramura K, Sougawa H, Yada T, Wayner MJ, Sasaki K (2006) Leptin facilitates learning and memory performance and enhances hippocampal CA1 long-term potentiation and CaMK II phosphorylation in rats. Peptides 27:2738-2749

30. Lieb W, Beiser AS, Vasan RS, Tan ZS, Au R, Harris TB, Roubenoff R, Auerbach S, DeCarli C, Wolf PA, Seshadri S (2009) Association of plasma leptin levels with incident Alzheimer disease and MRI measures of brain aging. JAMA 302:2565-2572

31. Janssen I, Heymsfield SB, Ross R (2002) Low relative skeletal muscle mass (sarcopenia) in older persons is associated with functional impairment and physical disability. J Am Geriatr Soc 50:889-896
32. Canon ME, Crimmins EM (2011) Sex differences in the association between muscle quality, inflammatory markers, and cognitive decline. J Nutr Health Aging 15:695-698

33. Hsu YH, Liang CK, Chou MY, Liao MC, Lin YT, Chen LK, Lo YK (2014) Association of cognitive impairment, depressive symptoms and sarcopenia among healthy older men in the veterans retirement community in southern Taiwan: a cross-sectional study. Geriatr Gerontol Int 14:102-108

34. Nangle MR, Manchery N (2020) Can chronic oral inflammation and masticatory dysfunction contribute to cognitive impairment? Curr Opin Psychiatry 33:156-162

Publisher's Note Springer Nature remains neutral with regard to jurisdictional claims in published maps and institutional affiliations. 